\title{
Optical spectroscopy and the UV luminosity function of galaxies in the Abell 1367, Coma and Virgo clusters ${ }^{\star}$
}

\author{
L. Cortese ${ }^{1}$, G. Gavazzi ${ }^{1}$, J. Iglesias-Paramo ${ }^{2}$, A. Boselli ${ }^{2}$, and L. Carrasco ${ }^{3,4}$ \\ 1 Universitá degli Studi di Milano-Bicocca, P.zza della Scienza 3, 20126 Milano, Italy \\ e-mail: Giuseppe.Gavazzi@mib.infn.it \\ 2 Laboratoire d'Astrophysique de Marseille, BP8, Traverse du Siphon, 13376 Marseille, France \\ e-mail: jorge.iglesias@oamp.fr; alessandro.boselli@oamp.fr \\ 3 Instituto Nactional de Astrofisica, Optica y Electronica, Apartado Postal 51 C.P. 72000 Puebla, Pue., Mexico \\ e-mail: carrasco@transum.inaoep.mx \\ ${ }^{4}$ Observatorio Astronomico Nacional/UNAM, Ensenada B.C., Mexico
}

Received 12 November 2002 / Accepted 14 January 2003

\begin{abstract}
Optical spectroscopy of 93 galaxies, 60 projected in the direction of Abell 1367, 21 onto the Coma cluster and 12 on Virgo, is reported. The targets were selected because they were detected in previous H $\alpha$, UV or $r^{\prime}$ surveys. The present observations bring to $100 \%$ the redshift completeness of $\mathrm{H} \alpha$ selected galaxies in the Coma region and to $75 \%$ in Abell 1367. All observed galaxies except one show $\mathrm{H} \alpha$ emission and belong to the clusters. This confirms previous determinations of the $\mathrm{H} \alpha$ luminosity function of the two clusters that were based on the assumption that all $\mathrm{H} \alpha$ detected galaxies were cluster members. Using the newly obtained data we re-determine the UV luminosity function of Coma and we compute for the first time the UV luminosity function of A1367. Their faint end slopes remain uncertain $(-2.00<\alpha<-1.35)$ due to insufficient knowledge of the background counts. If $90 \%$ of the UV selected galaxies without redshift will be found in the background (as our survey indicates), the slope of UV luminosity function will be $\alpha \sim-1.35$, in agreement with the UV luminosity function of the field (Sullivan et al. 2000) and with the $\mathrm{H} \alpha$ luminosity functions of the two clusters (Iglesias-Paramo et al. 2002). We discover a point-like $\mathrm{H} \alpha$ source in the Virgo cluster, associated with the giant galaxy VCC873, possibly an extragalactic HII region similar to the one recently observed in Virgo by Gerhard et al. (2002).
\end{abstract}

Key words. galaxies: distances and redshifts - galaxies: luminosity function, mass function - galaxies: clusters: individuals: Abell 1367, Coma, Virgo

\section{Introduction}

Multi-wavelength determinations of the luminosity function of galaxies belonging to nearby rich clusters are fundamental tools for shedding light on the process of galaxy evolution and, in conjunction with similar determinations of "field" galaxies, should help unveil the role of the environment on galaxy evolution.

These determinations suffer from insufficient redshift coverage, a crucial parameter for discriminating the (minority of) cluster members from the background interlopers.

Luminosity functions based on narrow band (i.e. $\mathrm{H} \alpha$ ) surveys are less affected by the redshift incompleteness: only

Send offprint requests to: L. Cortese,

e-mail: Luca.Cortese@mib.infn.it

^ Based on observations obtained with the Loiano telescope belonging to the University of Bologna (Italy), with the G. Haro telescope of the INAOE (Mexico) and with the Calar Alto observatory operated by the Centro Astronomico Hispano Aleman (Spain). emission-line galaxies in a narrow recessional velocity range are detected by the limited band-width of the adopted filters. Complementary redshift information is nevertheless highly recommended in order to reject background objects whose $\mathrm{H} \beta$ or [OIII] lines would fall into the band designed to contain the redshifted $\mathrm{H} \alpha$ line. Iglesias-Paramo et al. (2002) carried out a deep $\mathrm{H} \alpha$ imaging survey of $1 \times 1$ degree area in Coma and A1367 clusters and obtained the first $\mathrm{H} \alpha$ luminosity function of nearby clusters of galaxies. At that time, approximately fifty percent of $\mathrm{H} \alpha$ emitting galaxies had no estimate of their recessional velocity. With the aim of measuring the remaining redshifts and to confirm the $\mathrm{H} \alpha$ emission of these galaxies, we carried out the spectroscopic survey presented in this paper. We also obtained redshifts of several UV-selected galaxies in the Coma and A1367 regions which enable us to re-compute the UV luminosity functions of the two clusters.

The present paper is arranged as follows: Sect. 2 contains a description of the galaxy selection criteria. The observations and the data-reduction procedures are described in Sect. 3. 
The new redshifts are presented in Sect. 4 along with the data on one interesting object in the Virgo cluster. The $\mathrm{H} \alpha$ and UV luminosity functions of A1367 and Coma are discussed in Sect. 5. Our conclusions are briefly summarized in Sect. 6.

\section{The sample selection criteria}

Galaxies in the present study were primarily selected among objects projected in the direction of the Abell 1367 and Coma clusters with $\mathrm{H} \alpha$ emission detected in the INT $\mathrm{H} \alpha$ survey of Iglesias-Paramo et al. (2002) or selected from the $r^{\prime}$ survey by Iglesias-Paramo et al. (2003). The $\mathrm{H} \alpha$ selected galaxies in the Coma cluster were all spectroscopically measured, while only $75 \%$ of them were observed in A1367. The $r^{\prime}$ selected galaxies with $r^{\prime} \leq 17$ have been observed with a completeness of $64 \%$ and $92 \%$ for A1367 and Coma respectively.

In addition to these, we selected 20 targets with UV magnitude $m_{\mathrm{uv}}<18.5$ detected by the FOCA balloon-borne wide field UV camera $(\lambda=2000 \AA ; \Delta \lambda=150 \AA$ ) (Donas et al. 1995 and Donas, private communication). The UV magnitude is defined as: $m_{\mathrm{uv}}=-2.5 \log \left(F_{2000}\right)-21.175$, where $F_{2000}$ is the flux in units of erg cm $\mathrm{cm}^{-2} \mathrm{~s}^{-1} \AA^{-1}$. The spatial resolution of the UV data is 20 arcsec FWHM (Milliard et al. 1992). The astrometric accuracy of these data is therefore typically $3-$ 5 arcsec, insufficient to unambiguously identify spectroscopic targets and discriminate between stars and galaxies. To overcome this limitation, we cross-correlated the FOCA catalogues of A1367 and Coma with the $r^{\prime}$ band catalogue of galaxies $\left(r^{\prime}<21\right)$ by Iglesias-Paramo et al. (2003), using a matching radius of 20 arcsec. In cases of multiple identifications we select the galaxy closest to the UV position. Including the present observations, spectra are available for $64 \%$ and $56 \%$ of the UV-selected galaxies with an optical counterpart in A1367 and Coma respectively.

A dozen faint $\mathrm{H} \alpha$-emitting galaxies in the Virgo cluster with no estimate of the recessional velocity in the literature were also selected as filler objects. These were serendipitously found around bright VCC galaxies by visual inspection of the net $(\mathrm{H} \alpha+[\mathrm{NII}])$ frames obtained at the INT by Boselli et al. (2002).

\section{Observations and data reduction}

Long-slit, low dispersion spectra of 93 galaxies were obtained in several observing runs since 2001 using the imaging spectrograph BFOSC attached to the Cassini $1.5 \mathrm{~m}$ telescope at Loiano (Italy), LFOSC at the $2.1 \mathrm{~m}$ telescope of the Guillermo Haro Observatory at Cananea (Mexico), and with CAFOS attached to the $2.2 \mathrm{~m}$ telescope of the Calar Alto Observatory (Spain). Table 1 lists the characteristics of the instrumentation in the adopted set-up.

The observations at Loiano (44 galaxies) were performed using a 2.0 or 2.5 arcsec slit, depending on the seeing conditions, generally oriented E-W. The wavelength calibration was secured with exposures of HeAr lamps. The on-target exposure time ranged between 15 and 30 min according to the brightness of the targets.
The observations at Cananea (40 galaxies) were carried out with a 1.9 arcsec slit, generally oriented $\mathrm{N}-\mathrm{S}$. The wavelength calibration was secured with exposures of XeNe lamps. The on-target exposure time ranged between 20 and 40 min according to the brightness of the targets. The observations at Calar Alto (9 galaxies) were carried out with a 1.5 arcsec slit, generally oriented $\mathrm{N}-\mathrm{S}$. The wavelength calibration was secured with exposures of $\mathrm{CdHe}$ lamps. The on-target exposure time ranged between 15 and 30 min according to the brightness of the targets. In all runs the observations were obtained in nearly photometric conditions, with thin cirrus. The orientation of the slit was modified from the set-up given above when two adjacent objects could be observed in the same exposure.

The data reduction was performed in the IRAF environment. After bias subtraction, when 3 or more frames of the same target were obtained, these were combined (after spatial alignment) using a median filter to help cosmic rays removal. Otherwise the cosmic rays were removed using the task COSMICRAYS and/or by visual inspection. The lamp wavelength calibration was checked against known sky lines. These were found within $\sim 1 \AA$ from their nominal position, providing an estimate of the systematic uncertainty on the derived velocity of $\sim 50 \mathrm{~km} \mathrm{~s}^{-1}$. After subtraction of sky background, one-dimensional spectra were extracted from the frames. The redshift were obtained using the cross-correlation technique of Tonry \& Davis (1979). This method is based on a "comparison" between the spectrum of a galaxy (or a star) whose redshift is to be determined, and a fiducial spectral template of a galaxy of the appropriate spectral type to contain the wanted absorption/emission lines. The basic assumption behind this method is that the spectrum of a galaxy is well approximated by the spectrum of its stars, modified by the effects of the stellar motion inside the galaxy and by the systemic redshift. For this purpose, high signal-to-noise spectra were taken of two template galaxies: VCC0066 (emission lines) and NGC 221 (absorption lines), which were converted to the rest frame $\lambda$. The derived redshifts are not transformed to heliocentric.

\section{Results}

The velocity measurements obtained in this work are listed in Table 2 as follow:

Column 1: Galaxy designation. For Virgo cluster we use the VCC (Binggeli et al. 1985) and VPC (Young \& Currie 1998) designations when available.

Columns 2, 3: (J2000) celestial coordinates, measured with a few arcsec uncertainty.

Column 4: $r^{\prime}$ band magnitude.

Columns 5, 6: Observed recessional velocity with uncertainty derived in this work. The latter quantity includes only statistical errors. The global uncertainty can be derived by adding in quadrature of $50 \mathrm{~km} \mathrm{~s}^{-1}$ due to the uncertainty in the absolute wavelength calibration.

Column 7: Type of lines ( $\mathrm{A}=$ absorption; $\mathrm{E}=$ Emission $)$.

Column 8: Telescope $(\mathrm{LOI}=$ Loiano; $\mathrm{CAN}=$ Cananea, $\mathrm{CAL}=$ Calar Alto).

Column 9: Galaxy selection band (UV, $\mathrm{H} \alpha$ or $r^{\prime}$ ). 
Table 1. The spectrograph characteristics.

\begin{tabular}{lccccc}
\hline \hline Observatory & Spectrograph & $\begin{array}{c}\text { Dispersion } \\
\AA / \mathrm{mm}\end{array}$ & $\begin{array}{c}\text { Coverage } \\
\AA\end{array}$ & CCD & $\begin{array}{c}\text { pix } \\
\mu \mathrm{m}\end{array}$ \\
\hline Loiano & BFOSC & 198 & $3600-8900$ & $1340 \times 1300$ EEV & 20 \\
Cananea & LFOSC & 228 & $4000-7100$ & $576 \times 384$ TH & 23 \\
Calar Alto & CAFOS & 187 & $3600-10200$ & $2048 \times 2048$ SIT & 24 \\
\hline
\end{tabular}

\subsection{The Virgo cluster}

The twelve redshift measurements presented in this work are of faint $\left(r^{\prime} \leq 18\right)$ objects in the Virgo cluster that were serendipitously detected near bright VCC galaxies as part of an $\mathrm{H} \alpha$ imaging survey of this cluster (Boselli et al. 2002). Four of these galaxies turned out to be background galaxies whose emission, revealed in the net $(\mathrm{H} \alpha+[\mathrm{NII}])$ frames, is in fact due to $\mathrm{H} \beta$ and [OIII] lines, showing that at the faint limit of the survey the contamination by this type of object becomes relevant.

We would like to draw attention to the point-like $\mathrm{H} \alpha$ source $122603+130724$ detected at the projected distance of $2^{\prime}$ from the giant galaxy VCC873 shown in Fig. 1. The source shows an $\mathrm{H} \alpha$ equivalent width of $\sim 700 \AA$ and an $\mathrm{H} \alpha$ flux $\sim 10^{-14.6} \mathrm{erg} \mathrm{s}^{-1} \mathrm{~cm}^{-2}$. In spite of its very faint continuum $\left(r^{\prime} \sim 21.5\right)$, the $15 \mathrm{~min}$ spectrum of this object taken with the Loiano $1.5 \mathrm{~m}$ telescope yielded a prominent $\mathrm{H} \alpha$ emission at $260 \mathrm{~km} \mathrm{~s}^{-1}$, consistent with the velocity of VCC873 $\left(232 \mathrm{~km} \mathrm{~s}^{-1}\right)$.

Unlike planetary nebulae (ICPNe) found near giant galaxies in the Virgo and Fornax cluster (Arnaboldi et al. 1996; Theuns \& Warren 1997) showing strong [OIII] $15007 \AA$ lines (Dopita et al. 1992), 122603+130724 is not detected in [OIII]. However, given the weakness of the available spectrum, the upper limit on [OIII] is $\sim 0.3$ of the $\mathrm{H} \alpha$ flux, therefore consistent with $122603+130724$ being an extragalactic HII region (Dopita et al. 2000) associated with the giant galaxy VCC873. The point-like $\mathrm{H} \alpha$ source $122544+130740$ listed in Table 2 could be another extragalactic HII region, in this case not associated with a bright cluster galaxy. However the redshift of this faint $\left(r^{\prime} \sim 20\right)$ object, based on one line (presumably $\mathrm{H} \alpha$ ), needs confirmation because it lies at a projected distance of $10 \operatorname{arcsec}$ from a bright star.

Gerhard et al. (2002) found 17 candidate extragalactic HII regions in the Virgo cluster, among which the one associated with VCC836 was confirmed spectroscopically. Objects of this kind could account for the diffuse intracluster light (Bernstein et al. 1995) and might contribute to the enrichment of the intergalactic medium in clusters.

\section{The luminosity functions}

\subsection{The Ha luminosity function}

With the new data presented in this work, the redshift of all $\mathrm{H} \alpha$ selected galaxies in the Coma cluster (Iglesias-Paramo et al. 2002) have been measured. All objects turned out to be cluster members $\left(4000<V<10000 \mathrm{~km} \mathrm{~s}^{-1}\right)$ and significant $\mathrm{H} \alpha$ emission was detected in their spectra. The $\mathrm{H} \alpha$ luminosity function

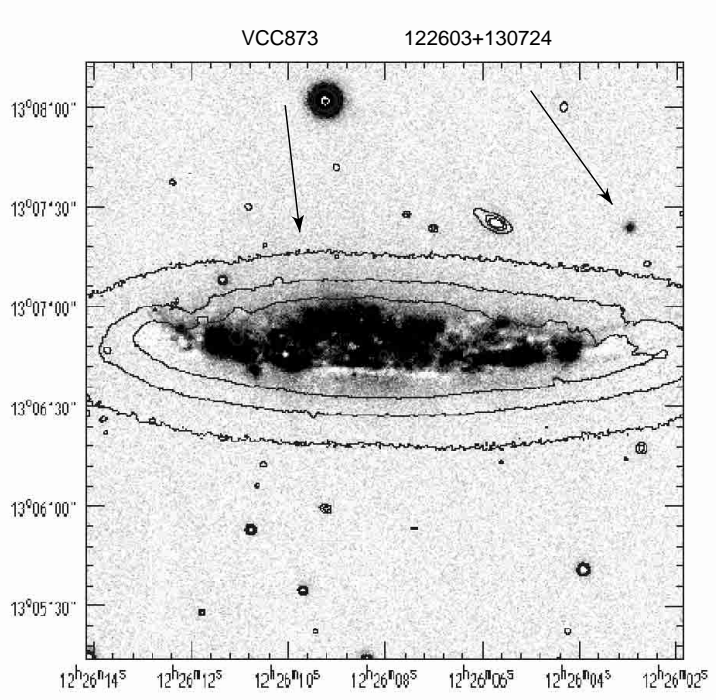

Fig. 1. Net $\mathrm{H} \alpha+[\mathrm{NII}]$ image of $122603+130724$ and VCC873 (greyscale) with superposed contours of the continuum emission.

determined by Iglesias-Paramo et al. (2002) assuming that all objects were at the distance of Coma is fully confirmed.

In A 1367 we measured the redshift of 14 galaxies, bringing to $75 \%$ the completeness of $\mathrm{H} \alpha$ selected galaxies in this cluster. The membership of the cluster was confirmed for 13 galaxies, while $1(114430+195718)$ turned out to be a background galaxy whose emission, revealed by Iglesias-Paramo et al. (2002), is in fact due to $\mathrm{H} \beta$ and [OIII] lines. This confirms that the $\mathrm{H} \alpha$ survey by Iglesias-Paramo et al. (2002) is little contaminated by $\mathrm{H} \beta$ and [OIII] emission lines from background objects, emphasizing the high success-rate of selecting emission line members with the Iglesias-Paramo et al. (2002) method.

\subsection{The UV luminosity function}

The H $\alpha$ luminosity functions of Coma and A1367 are found consistent one another, being characterized by a faint end slope $\alpha \sim-0.7$. This value differs significantly from the slope of the UV luminosity function of Coma (Andreon 1999, hereafter A99) $\alpha \sim-2.0$, the only UV luminosity function ever determined for a cluster. While stars with masses $M>10 M_{\odot}$ and lifetimes $<20 \mathrm{Myr}$ contribute significantly to the $\mathrm{H} \alpha$ flux, the UV emission is dominated by young stars of intermediate masses $\left(2<M<5 M_{\odot}\right)$. Furthermore, UV emission 
Table 2. Spectroscopic parameters of the observed galaxies.

\begin{tabular}{|c|c|c|c|c|c|c|c|c|}
\hline Name & $\begin{array}{c}\text { RA } \\
(\mathrm{J} .2000)\end{array}$ & $\begin{array}{c}\text { Dec } \\
(\mathrm{J} .2000)\end{array}$ & $\begin{array}{c}r^{\prime} \\
\operatorname{mag}\end{array}$ & $\begin{array}{c}\text { vel } \\
\mathrm{km} \mathrm{s}^{-1}\end{array}$ & \pm & Lines & Tel. & Sel. \\
\hline \multicolumn{9}{|c|}{ A1367 } \\
\hline $114038+195437$ & 114038.96 & +195437.4 & 17.48 & 7784 & 96 & $\mathrm{E}$ & LOI & $\mathrm{H} \alpha$ \\
\hline $114110+201117$ & 114110.47 & +2011 17.7 & 17.57 & 6952 & 22 & $\mathrm{E}$ & LOI & $\mathrm{H} \alpha$ \\
\hline $114117+200832$ & 114117.60 & +200832.0 & 15.93 & 14500 & 20 & $\mathrm{E}$ & LOI & UV \\
\hline $114137+194451$ & 114137.90 & +194451.0 & 17.14 & 27891 & 80 & A & CAN & UV \\
\hline $114141+200230$ & 114141.20 & +200230.5 & 17.37 & 8447 & 51 & $\mathrm{E}$ & LOI & $\mathrm{H} \alpha$ \\
\hline $114142+200054$ & 114142.57 & +200054.9 & 17.33 & 8456 & 69 & $\mathrm{E}$ & LOI & $\mathrm{H} \alpha$ \\
\hline $114149+194605$ & 114149.79 & +194605.1 & 17.52 & 7789 & 39 & $\mathrm{E}$ & LOI & $\mathrm{H} \alpha$ \\
\hline $114156+194836$ & 114157.03 & +194836.2 & 16.53 & 28896 & 140 & A & CAN & $r^{\prime}$ \\
\hline $114229+195238$ & 114229.00 & +195238.0 & 17.37 & 14084 & 130 & A & CAN & UV \\
\hline $114238+192103$ & 114238.40 & +192103.0 & 17.16 & 18674 & 72 & $\mathrm{E}$ & LOI & UV \\
\hline $114240+195716$ & 114240.36 & +195716.6 & 17.68 & 7501 & 46 & $\mathrm{E}$ & LOI & $\mathrm{H} \alpha$ \\
\hline $114252+192543$ & 114252.22 & +192543.4 & 15.13 & 24452 & 114 & A & LOI & $r^{\prime}$ \\
\hline $114253+201039$ & 114253.11 & +201039.8 & 15.43 & 6261 & 192 & A & CAN & $r^{\prime}$ \\
\hline $114300+201225$ & 114300.30 & +201225.9 & 14.90 & 20965 & 133 & A & CAN & $r^{\prime}$ \\
\hline $114306+195620$ & 114306.31 & +195620.0 & 15.21 & 6321 & 103 & A & LOI & $r^{\prime}$ \\
\hline $114308+194155$ & 114308.00 & +194155.0 & 16.45 & 12988 & 16 & $\mathrm{E}$ & LOI & UV \\
\hline $114311+200144$ & 114311.19 & +200144.9 & 15.37 & 6994 & 102 & A & CAN & $r^{\prime}$ \\
\hline $114312+193841$ & 114312.68 & +193841.0 & 16.23 & 27884 & 152 & A & CAN & $r^{\prime}$ \\
\hline $114313+193645$ & 114313.08 & +193645.8 & 17.27 & 6121 & 131 & $\mathrm{E}$ & LOI & $\mathrm{H} \alpha$ \\
\hline $114314+194016$ & 114314.23 & +194016.7 & 15.03 & 28078 & 142 & A & CAN & $r^{\prime}$ \\
\hline $114315+195614$ & 114315.57 & +195615.3 & 16.57 & 28847 & 117 & A & CAN & $r^{\prime}$ \\
\hline $114331+200058$ & 114331.30 & +200058.0 & 16.44 & 30164 & 23 & $\mathrm{E}$ & LOI & UV \\
\hline $114337+191836$ & 114337.70 & +191836.0 & 16.65 & 24154 & 132 & $\mathrm{E}$ & CAN & UV \\
\hline $114341+200135$ & 114341.62 & +200135.3 & 17.08 & 6455 & 66 & $\mathrm{E}$ & LOI & $\mathrm{H} \alpha$ \\
\hline $114345+192332$ & 114345.41 & +192332.0 & 15.60 & 27889 & 258 & A & LOI & $r^{\prime}$ \\
\hline $114350+192606$ & 114350.85 & +192606.2 & 15.31 & 6135 & 141 & A & CAN & $r^{\prime}$ \\
\hline $114353+194321$ & 114353.88 & +194321.3 & 17.15 & 24613 & 185 & A & CAN & $r^{\prime}$ \\
\hline $114353+194428$ & 114353.77 & +194428.6 & 15.30 & 6120 & 131 & A & CAN & $r^{\prime}$ \\
\hline $114355+192743$ & 114355.71 & +192743.9 & 18.72 & 6427 & 50 & $\mathrm{E}$ & LOI & $\mathrm{H} \alpha$ \\
\hline $114357+195607$ & 114357.46 & +195607.6 & 15.47 & 7057 & 88 & A & CAN & $r^{\prime}$ \\
\hline $114401+191555$ & 114401.10 & +19 1555.0 & 16.57 & 28353 & 76 & A & CAN & UV \\
\hline $114401+191707$ & 114401.00 & +191707.0 & 16.92 & 12784 & 91 & $\mathrm{E}$ & CAN & UV \\
\hline $114401+192953$ & 114401.00 & +192953.0 & 16.31 & 19367 & 25 & $\mathrm{E}$ & LOI & UV \\
\hline $114403+194433$ & 114403.31 & +194433.0 & 15.53 & 6715 & 101 & A & CAN & $r^{\prime}$ \\
\hline $114407+193724$ & 114407.80 & +193724.0 & 17.28 & 39774 & 85 & A & CAN & UV \\
\hline $114413+192012$ & 114413.80 & +192012.0 & 16.90 & 5852 & 47 & $\mathrm{E}$ & LOI & $r^{\prime}$ \\
\hline $114419+191902$ & 114419.30 & +191902.0 & 16.90 & 26091 & 217 & $\mathrm{E}$ & CAN & UV \\
\hline $114430+195718$ & 114430.41 & +195718.8 & 20.23 & 96341 & 19 & $\mathrm{E}$ & LOI & $\mathrm{H} \alpha$ \\
\hline $114444+194814$ & 114444.28 & +194814.0 & 19.62 & 8098 & 62 & $\mathrm{E}$ & CAL & $\mathrm{H} \alpha$ \\
\hline $114446+194737$ & 114446.13 & +194737.5 & 19.35 & 8240 & 59 & $\mathrm{E}$ & CAL & $\mathrm{H} \alpha$ \\
\hline $114446+194639$ & 114446.68 & +194639.5 & 22.03 & 8383 & 36 & $\mathrm{E}$ & CAL & $\mathrm{H} \alpha$ \\
\hline $114447+194648$ & 114447.54 & +194648.8 & 21.81 & 8428 & 33 & $\mathrm{E}$ & CAL & $\mathrm{H} \alpha$ \\
\hline $114450+194605$ & 114450.81 & +194605.1 & 20.16 & 8089 & 50 & $\mathrm{E}$ & LOI & $\mathrm{H} \alpha$ \\
\hline $114451+194717$ & 114451.27 & +194717.5 & 19.24 & 8022 & 35 & $\mathrm{E}$ & LOI & $\mathrm{H} \alpha$ \\
\hline $114454+194733$ & 114454.22 & +194733.2 & 18.32 & 8067 & 31 & $\mathrm{E}$ & LOI & $\mathrm{H} \alpha$ \\
\hline $114454+200101$ & 114454.55 & +200102.0 & 16.14 & 7646 & 500 & $\mathrm{E}$ & CAN & $\mathrm{H} \alpha$ \\
\hline $114459+194757$ & 114459.35 & +194757.1 & 18.42 & 39550 & 79 & $\mathrm{E}$ & LOI & $r^{\prime}$ \\
\hline $114501+194550$ & 114501.90 & +194550.0 & 16.74 & 20575 & 30 & $\mathrm{E}$ & CAN & UV \\
\hline $114502+194520$ & 114502.71 & +194520.6 & 16.74 & 20626 & 13 & $\mathrm{E}$ & LOI & $r^{\prime}$ \\
\hline
\end{tabular}


Table 2. continued.

\begin{tabular}{|c|c|c|c|c|c|c|c|c|}
\hline Name & $\begin{array}{c}\text { RA } \\
(\mathrm{J} .2000)\end{array}$ & $\begin{array}{c}\text { Dec } \\
(\mathrm{J} .2000)\end{array}$ & $\begin{array}{c}r^{\prime} \\
\mathrm{mag}\end{array}$ & $\begin{array}{c}\mathrm{vel} \\
\mathrm{km} \mathrm{s}^{-1}\end{array}$ & \pm & Lines & Tel. & Sel. \\
\hline $114505+194514$ & 114505.67 & +194514.8 & 18.07 & 20263 & 150 & A & CAN & $r^{\prime}$ \\
\hline $114506+194733$ & 114505.98 & +194733.6 & 16.82 & 19919 & 58 & $\mathrm{E}$ & LOI & $r^{\prime}$ \\
\hline $114506+200921$ & 114506.56 & +200921.4 & 15.36 & 6145 & 184 & A & LOI & $r^{\prime}$ \\
\hline $114513+194523$ & 114513.77 & +194522.2 & 15.84 & 8316 & 224 & $\mathrm{E}$ & CAN & $\mathrm{H} \alpha$ \\
\hline $114514+200836$ & 114514.10 & +200836.0 & 16.99 & 3752 & 43 & $\mathrm{E}$ & CAN & UV \\
\hline $114518+200009$ & 114518.00 & +200009.5 & 17.54 & 5327 & 28 & $\mathrm{E}$ & LOI & $\mathrm{H} \alpha$ \\
\hline $114525+194905$ & 114525.56 & +194905.7 & 15.22 & 8422 & 123 & A & $\mathrm{CAN}$ & $r^{\prime}$ \\
\hline $114541+194613$ & 114541.16 & +194613.4 & 14.89 & 5419 & 138 & A & CAN & $r^{\prime}$ \\
\hline $114545+194130$ & 114545.20 & +194130.0 & 16.48 & 6123 & 17 & $\mathrm{E}$ & CAN & UV \\
\hline $114558+194810$ & 114558.59 & +1948 10.9 & 15.41 & 5493 & 173 & A & LOI & $r^{\prime}$ \\
\hline $114611+195110$ & 114611.99 & +1951 10.0 & 15.07 & 5441 & 101 & A & CAN & $r^{\prime}$ \\
\hline \multicolumn{9}{|c|}{ Coma } \\
\hline $125802+282722$ & 125802.10 & +282722.0 & 17.01 & 48300 & 150 & A & CAN & UV \\
\hline $125815+283117$ & 125815.12 & +283117.2 & 16.70 & 6725 & 56 & $\mathrm{E}$ & LOI & UV \\
\hline $125823+281945$ & 125823.66 & +281945.6 & 16.26 & 8352 & 126 & A & CAN & $r^{\prime}$ \\
\hline $125828+283135$ & 125828.45 & +283135.9 & 15.24 & 18290 & 104 & A & CAN & $r^{\prime}$ \\
\hline $125829+281806$ & 125829.44 & +281806.3 & 15.52 & 6082 & 99 & A & CAN & $r^{\prime}$ \\
\hline $125845+283235$ & 125845.80 & +283235.3 & 17.76 & 6333 & 14 & $\mathrm{E}$ & LOI & $\mathrm{H} \alpha$ \\
\hline $125845+284133$ & 125845.64 & +284133.1 & 17.21 & 6682 & 149 & $\mathrm{E}$ & LOI & $\mathrm{H} \alpha$ \\
\hline $125856+282749$ & 125856.19 & +28 2749.0 & 15.23 & 5927 & 156 & A & CAN & $r^{\prime}$ \\
\hline $125918+283726$ & 125917.90 & +283726.0 & 16.81 & 10340 & 32 & $\mathrm{E}$ & CAN & UV \\
\hline $125923+282918$ & 125923.06 & +282918.4 & 15.51 & 7015 & 165 & $\mathrm{E}$ & CAN & $\mathrm{H} \alpha$ \\
\hline $125924+282050$ & 125924.11 & +28 2050.1 & 16.62 & 20241 & 26 & $\mathrm{E}$ & LOI & UV \\
\hline $125941+283026$ & 125941.12 & +283026.6 & 15.45 & 8314 & 161 & A & CAN & $r^{\prime}$ \\
\hline $125956+275548$ & 125956.68 & +275548.2 & 15.31 & 7653 & 93 & A & CAN & $r^{\prime}$ \\
\hline $130004+283614$ & 130004.51 & +283614.1 & 15.32 & 6727 & 153 & A & CAN & $r^{\prime}$ \\
\hline $130037+283951$ & 130037.22 & +283951.7 & 16.62 & 7095 & 87 & $\mathrm{E}$ & CAN & $\mathrm{H} \alpha$ \\
\hline $130127+281053$ & 130127.29 & +28 1053.9 & 17.18 & 48821 & 100 & A & CAN & $r^{\prime}$ \\
\hline $130128+281515$ & 130128.63 & +281515.9 & 20.41 & 9061 & 33 & $\mathrm{E}$ & LOI & $\mathrm{H} \alpha$ \\
\hline $130130+283328$ & 130130.85 & +283328.0 & 16.76 & 6885 & 194 & $\mathrm{E}$ & LOI & $\mathrm{H} \alpha$ \\
\hline $130140+281456$ & 130140.97 & +281456.6 & 19.33 & 9286 & 20 & $\mathrm{E}$ & LOI & $\mathrm{H} \alpha$ \\
\hline $130151+280822$ & 130151.90 & +280822.0 & 17.00 & 28754 & 27 & A & CAL & UV \\
\hline $130158+282114$ & 130158.43 & +282114.8 & 19.81 & 8577 & 18 & $\mathrm{E}$ & LOI & $\mathrm{H} \alpha$ \\
\hline \multicolumn{9}{|c|}{ Virgo } \\
\hline VCC1715 & 123728.45 & +084739.2 & 15.37 & 888 & 32 & $\mathrm{E}$ & LOI & $\mathrm{H} \alpha$ \\
\hline VCC 404 & 122017.50 & +041210.0 & 14.20 & 1746 & 93 & $\mathrm{E}$ & LOI & $\mathrm{H} \alpha$ \\
\hline VPC766 & 123046.20 & +120557.1 & 17.05 & 1162 & 47 & $\mathrm{E}$ & LOI & $\mathrm{H} \alpha$ \\
\hline $122544+130740$ & 122544.20 & +130740.0 & 20.10 & 796 & 37 & $\mathrm{E}$ & CAL & $\mathrm{H} \alpha$ \\
\hline $122603+130723$ & 122602.91 & +130723.7 & 21.50 & 260 & 34 & $\mathrm{E}$ & LOI & $\mathrm{H} \alpha$ \\
\hline $122605+130725$ & 122605.75 & +130725.7 & 17.14 & 23980 & 40 & $\mathrm{E}$ & LOI & $r^{\prime}$ \\
\hline $123015+122812$ & 123015.20 & +122812.0 & 21.10 & 79521 & 37 & $\mathrm{E}$ & CAL & $\mathrm{H} \alpha$ \\
\hline $123021+121614$ & 123021.10 & +121614.0 & 20.70 & 90792 & 36 & $\mathrm{E}$ & CAL & $\mathrm{H} \alpha$ \\
\hline $123222+090944$ & 123222.81 & +090944.1 & 17.97 & 77812 & 50 & $\mathrm{E}$ & LOI & $\mathrm{H} \alpha$ \\
\hline $123315+091448$ & 123315.25 & +091448.0 & 17.59 & 30923 & 29 & $\mathrm{E}$ & LOI & $r^{\prime}$ \\
\hline $123728+082540$ & 123728.00 & +082540.0 & 20.60 & 79521 & 33 & $\mathrm{E}$ & CAL & $\mathrm{H} \alpha$ \\
\hline $124547+102620$ & 124547.32 & +102620.5 & 16.41 & 36946 & 144 & $\mathrm{E}$ & LOI & $r^{\prime}$ \\
\hline
\end{tabular}




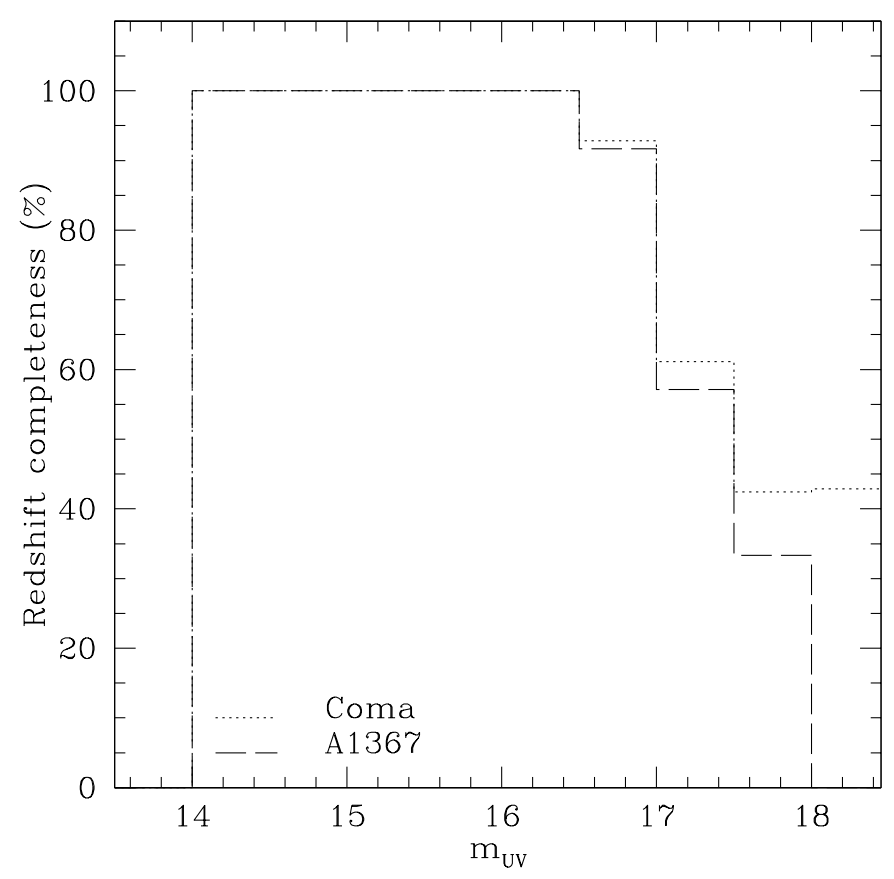

Fig. 2. Redshift completeness of the UV selected galaxies in Coma (dotted histogram) and A1367 (long dashed histogram). The redshift completeness is $\sim 90 \%$ for $m_{\mathrm{UV}} \leq 16.75$ while at fainter magnitudes it drops rapidly below $50 \%$.

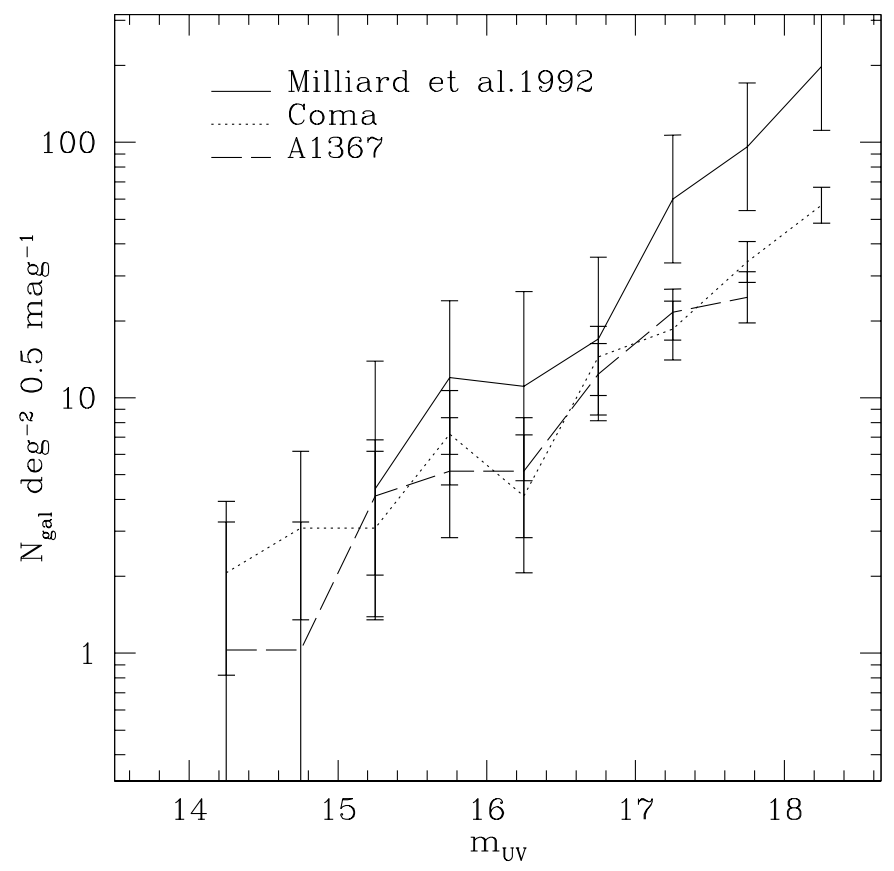

Fig. 3. Differential UV galaxy counts in the field by Milliard et al. 1992 (solid line), in the Coma (dotted line) and A1367 (long dashed line) clusters.

is detected also from early-type galaxies with no recent star formation episodes (Deharveng et al. 2002). However, these differences alone are insufficient to produce a discrepant luminosity function such as obtained in the two bands. Thus we use the spectroscopic observations presented in this work to recompute the Coma UV luminosity function and to compute, for

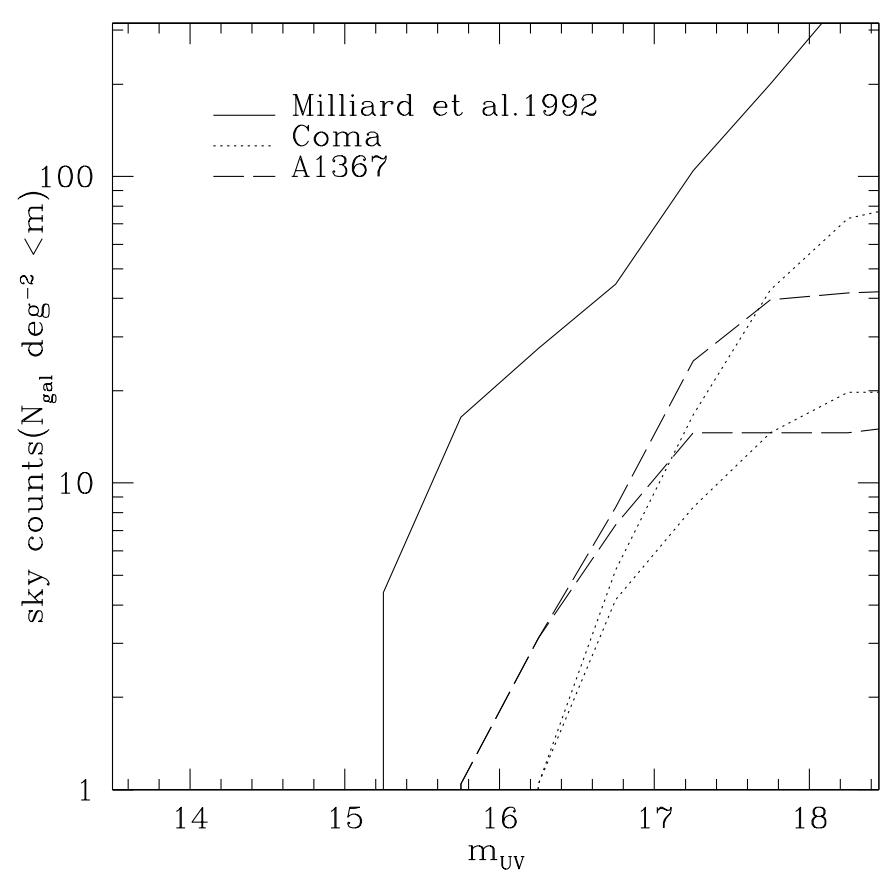

Fig. 4. Integrated UV galaxy counts in the field by Milliard et al. 1992 (solid line), upper and lower background limits for Coma (dotted line) and for A1367 (long dashed line) are given.

the first time, the UV luminosity function of A1367. Using the same UV data available to A99, we cross-correlate the UV detections with the $r^{\prime}$ catalogue of Iglesias-Paramo et al. (2003) for a better star/galaxy discrimination. This reduces our luminosity function determination to an area of $0.97^{\circ 2}$ (the same over which we determined the $\mathrm{H} \alpha$ luminosity function), instead of $\sim 3^{\circ 2}$ analyzed by A99. The determination of the cluster luminosity function requires a reliable estimate of the contribution of background/foreground objects to the UV counts. This can be accurately achieved for $m_{\mathrm{UV}} \leq 16.75$, since for these UV sources the redshift completeness is $\sim 90 \%$ (see Fig. 2). At fainter magnitudes the redshift completeness drops rapidly, thus requiring the contamination to be estimated statistically from the number of field galaxies, per bin of UV magnitude, that are expected to be randomly projected onto the cluster area. The over-density of UV counts due to Coma/A1367 galaxies can be derived from the difference between the counts in the direction of the two clusters and the UV galaxy counts derived in three random fields by Milliard et al. (1992) using the same experimental set up as for Coma and A1367. Opposite to our expectation, the cluster galaxy counts at faint magnitudes are $\sim 3$ times lower than the Milliard et al. (1992) number counts (see Fig. 3), as already noticed by A99. The reason for such a discrepancy is not fully understood and might reflect a higher degree of star contamination among the UV counts than estimated by Milliard et al. (1992). Alternatively it might derive from a higher than expected fraction of foreground galaxies in Milliard et al. (1992). However, foreground galaxies should contribute significantly less than background objects due to the small sampled volume at $V<4000 \mathrm{~km} \mathrm{~s}^{-1}$. Using our new redshifts we can at least estimate a minimum and a maximum 


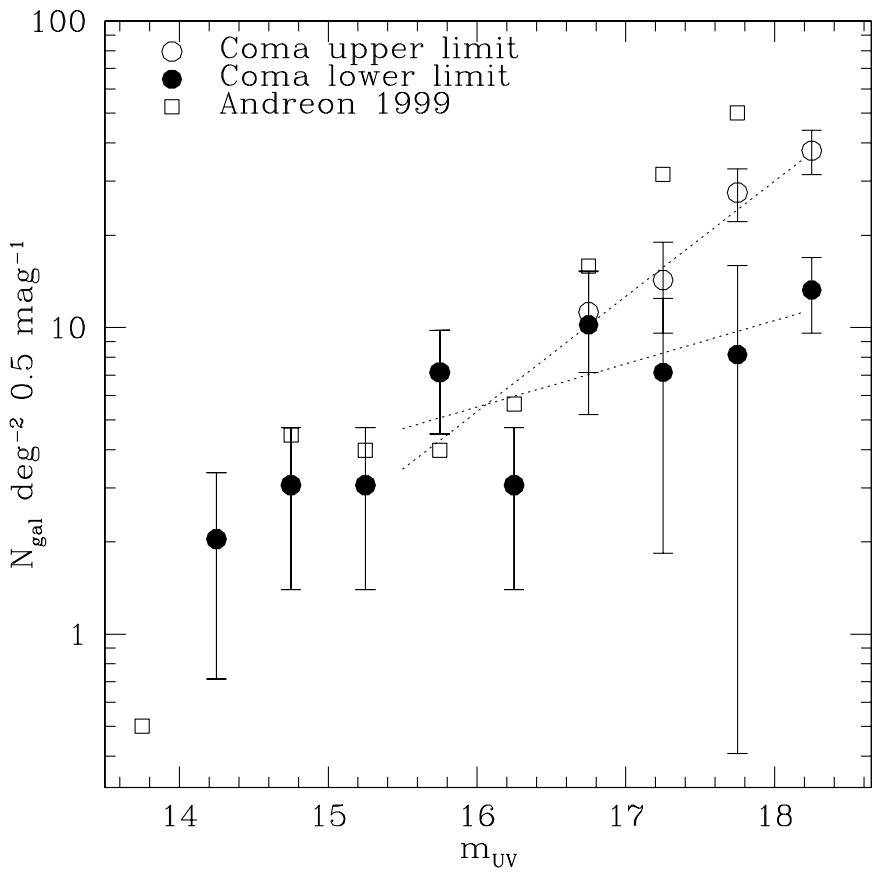

Fig. 5. The UV luminosity functions of the Coma galaxies. The luminosity function by A99 is represented with empty squares and the upper (empty circles) and lower (filled points) limits determined in this work are given. The dotted lines represent the best linear fits to the faint-end of the luminosity function. Error bars account for Poissonian fluctuations.

background contribution to the observed counts in the direction of Coma and A1367, a method followed by A99. The true background is at least composed of all objects whose velocity falls outside the assumed range for the clusters $(4000<V<$ $10000 \mathrm{~km} \mathrm{~s}^{-1}$ ). At most it comprises also all galaxies with unknown redshift. These limiting estimates, shown in Fig. 4 for Coma (dotted line) and A1367 (long dashed line), are found in good reciprocal agreement, but are significantly lower than the background counts estimated by Milliard et al. (1992). The resulting cluster UV luminosity functions should lie in between the two determinations given in Figs. 5 and 6.

Due to the large uncertainties we did not attempt to fit a complete Schechter (1976) function to the data. Instead we fit the low-end slope $\left(15.5<m_{\mathrm{UV}}<18\right)$ with an exponential form of slope $k m_{\mathrm{UV}}$ where $m_{\mathrm{UV}}$ is the UV magnitude and $k$ is related to the $\alpha$ parameter of the Schecther function by:

$\alpha=-(k / 0.4+1)$.

The resulting UV luminosity function of A1367 shows a slope between $k_{\text {lower }}=0.14 \pm 0.08$ and $k_{\text {upper }}=0.42 \pm 0.09$ (equivalent to $\alpha_{\text {lower }}=-1.35 \pm 0.20$ and $\alpha_{\text {upper }}=-2.05 \pm 0.22$ ). Similarly, for the UV luminosity function of Coma we find $k_{\text {lower }}=0.14 \pm 0.09$ and $k_{\text {upper }}=0.37 \pm 0.09$ (equivalent to $\alpha_{\text {lower }}=-1.35 \pm 0.22$ and $\left.\alpha_{\text {upper }}=-1.94 \pm 0.24\right)$. The upper and lower limits of the UV luminosity functions of the two clusters are in fair agreement, but the allowed range between the two limits is still very broad. Likely the low limit gives a more realistic representation of the true UV luminosity function because nearly all (18/20 or $90 \%)$ of the UV-selected galaxies for which we obtained a redshift in this work turned out to be in

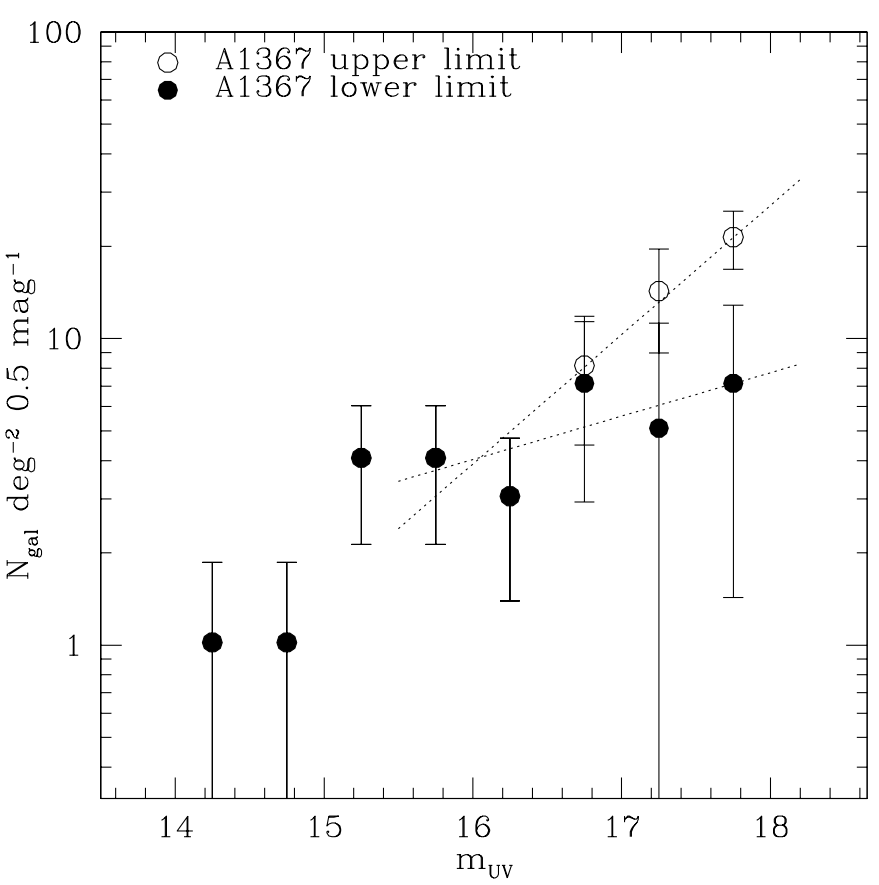

Fig. 6. Same as Fig. 5 for A1367.

the background. The low limit slope $\alpha \sim-1.35$ is in agreement with the field UV luminosity function of Sullivan et al. (2000) and it is consistent with the slope of the $\mathrm{H} \alpha$ luminosity function of the two clusters as determined by Iglesias-Paramo et al. (2002). Certainly the very steep $(\alpha \sim-2.0,-2.2)$ luminosity function found for Coma by A99 is due to an underestimate of the density of background galaxies.

\section{Summary and conclusions}

Optical spectroscopy of 93 galaxies, 60 of which are projected in the direction of Abell 1367, 21 onto the Coma cluster and 12 onto Virgo, is reported. These observations bring the redshift completeness among $\mathrm{H} \alpha$ selected galaxies by IglesiasParamo et al. (2002) to $100 \%$ in the Coma region and to $75 \%$ in Abell 1367. All, except one, of the $\mathrm{H} \alpha$ selected galaxies show $\mathrm{H} \alpha$ emission and are confirmed cluster members. The exception is a background galaxy whose [OIII] lines fall in the $\mathrm{H} \alpha$ filter. The $\mathrm{H} \alpha$ luminosity function of the two clusters determined by Iglesias-Paramo et al. (2002) is fully confirmed.

Redshifts of UV selected galaxies in Coma and A1367 regions were also obtained. With these new data the redshift completeness in the core of the two clusters has reached $\sim 90 \%$ for $m_{\mathrm{UV}} \leq 16.75$. We re-determine the Coma UV luminosity function and we compute for the first time the UV luminosity function of A1367. The two are found in fair agreement. Their faint-end slopes remain unconstrained $(-2.00<\alpha<-1.35)$ due to the uncertainty on the background counts. However, if $90 \%$ of the UV-selected galaxies without a redshift measurement will be found in the cluster background, as our data indicate, the slope of the UV luminosity function should be near $\alpha \sim-1.35$, in agreement with the field one (Sullivan et al. 2000) and with the $\mathrm{H} \alpha$ luminosity functions of the two clusters (Iglesias-Paramo et al. 2002). 
Finally we discover a point-like $\mathrm{H} \alpha$ source in the Virgo cluster, associated with the giant galaxy VCC873, possibly an extragalactic HII region like the one recently observed in Virgo by Gerhard et al. (2002). Objects of this kind could account for the diffuse intracluster light and might contribute to the enrichment of the intergalactic medium in galaxy clusters.

Acknowledgements. We wish to thank Jose Donas for providing us with his unpublished catalogue of UV sources in the Coma and Abell 1367 fields. The TACS of the Loiano, Cananea and Calar Alto telescopes are acknowledged for the generous time allocation to this project. This work could not have been completed without the NASA/IPAC Extragalactic Database (NED) which is operated by the Jet Propulsion Laboratory, Caltech under contract with NASA.

\section{References}

Andreon, S. 1999, A\&A, 351, 65

Arnaboldi, M., Freeman, K. C., Mendez, R. H., et al. 1996, ApJ, 472, 145

Bernstein, G. M., Nichol, R. C., Tyson, J. A., Ulmer, M. P., \& Wittman, D. 1995, AJ, 110, 1507
Binggeli, B., Sandage, A., \& Tammann, G. 1985, AJ, 90, 1681

Boselli, A., Iglesias-Paramo, J., Vilchez, J. M., \& Gavazzi, G. 2002, A\&A, 386, 134

Deharveng, J. M., Boselli, A., \& Donas, J. 2002, A\&A, 393, 843

Dopita, M. A., Jacoby, G. H., \& Vassiliadis, E. 1992, ApJ, 389, 27

Dopita, M. A., Kewley, L. J., Heisler, C. A., \& Sutherland, R. S. 2000, ApJ, 542, 224

Dressler, A. 1980, ApJ, 236, 351

Gerhard, O., Arnaboldi, M., Freeman, K. C., \& Okamura, S. 2002, ApJ, 580, L121

Iglesias-Paramo, J., Boselli, A., Cortese, L., Vilchez, J. M., \& Gavazzi, G. 2002, A\&A, 384, 383

Iglesias-Paramo, J., Boselli, A., Gavazzi, G., Cortese, L., \& Vilchez, J. M. 2003, A\&A, 397, 421

Milliard, B., Donas, J., Laget, M., Armand, C., \& Vuillemin, A. 1992, A\&A, 257, 24

Donas, J., Milliard, B., \& Laget, M. 1995, A\&A, 303, 661

Theuns, T., Warren, S. 1997, MNRAS, 284, L11

Tonry, J., \& Davis, M. 1979, AJ, 84, 1511

Schechter, P. 1976, ApJ, 203, 297

Sullivan, M., \& Treyer, M. A., Ellis, R. S., et al. 2000, MNRAS, 312, 442

Young, C. K., \& Currie, M. J. 1998, A\&AS, 127, 367 